\title{
Rights of Vulnerable People: Trembled in Health Care Setting
}

Shumaila Batool*and Santosh Kumar

School of Nursing and Midwifery, Aga Khan University, Karachi, Pakistan

*Corresponding author: Shumaila Batool, School of Nursing and Midwifery, Aga Khan University, Karachi, Pakistan, Tel: +92 3332715498; E-mail: Shumaila.batool.mn15@student.aku.edu

Received date: August 08, 2016; Accepted date: August 26, 2016; Published date: August 27, 2016

Copyright: $\odot 2016$ Batool S, et al. This is an open-access article distributed under the terms of the Creative Commons Attribution License, which permits unrestricted use, distribution, and reproduction in any medium, provided the original author and source are credited.

\begin{abstract}
Informed consent is obtained from a person undergoing any treatment and from surrogates if the person is not able to consent or is incompetent. In some cases where a person is incompetent and is unable to make consent then in such situations family decisions sometimes override individual's rights to autonomy, dignity and health care. This commentary article is based on a case study of a mentally retarded person whose family decided on his behalf for his organ donation to his brother. The aim of this paper is to critically review such cases, and attempts to provide an ethically sound decision in the identical cases.
\end{abstract}

Keywords: Organ donation; Transplantation; Inform consent; Mental retardation; Autonomy

\section{Introduction}

A surrogate or substitute decision maker is involved in medical decisions when an individual is incompetent to make his or her medical decisions. The question of who will serve as the surrogate and will decide on the behalf of an incompetent individual is ethical dilemmas which oblige court to reach at decisions. A big legal-ethical dilemma arises when the organ of an incompetent is sought to be for transplantation which must be explored under legal and ethical domains and should identify and appoint the authorities who take neutral decisions on their behalf. This paper is based on a clinical scenario of an incompetent individual who was selected for organ donation as a result of his family decision.

\section{Scenario}

In Surgical Intensive Care Unit of a private hospital, I was assigned with a liver donor patient. Post operatively, patient was on ventilator, and when it was planned to off the sedations and wean off the patient from ventilator, I observed that patient was not obeying my commands. I assumed that it could be due to the effects of sedation or language barrier. So I called patient's family to facilitate me in explaining the patient to cooperate with me and team so that he can easily get out of the ventilator. When family arrived, I explained to them that patient is not cooperating with me in weaning process. At that moment, I came to know that the liver donor patient was mentally retarded. On inquest, they revealed that no one in the family was ready to become a donor, so with the consensus of family it was decided to use him as a liver donor, as he is mentally retarded and have no quality of life.

Mental Retardation (MR) is defines as: "a state of slow or seized in the development course and an inadequate development of the mind, which is specially described by inefficiency in skills and social functioning, and which contributes in overall level of intelligence" [1].

Before, reflecting upon the above case scenario let us, start by posing the relevant questions. Do vulnerable people have less or limited rights in organ donations? Is it fair to involve them in organ donation procedure, if it is not their right to receive the organ transplantation? If so, then how the procedures of voluntariness inform consent and the principle of justice are being justified. As health care professionals, what we study and know about the Informed consent, "Is the procedure that must be before any health care intervention, which is derived from the principle of autonomy" [2]. It is an important component, which must not be avoided before initiation of any medical intervention [3]. Depending on the level of procedure, consent may be verbal or a written one. The purpose of this paper is to reflect on the reasons for the differences in theory and practice, and finding justification based on ethical grounds.

\section{My Position}

In health care settings, consent is obtained from the person who underwent treatment or procedure. It is individual's autonomous right to take decisions for their health and treatment. According to a recent Consensus Statement on Live Organ Donors: "the person who is live donor should be competent, and voluntarily without any oppression, and should be fully aware about the risks and benefits associated with the procedure" [4]. At one side, MR is considered as, contraindication for organ transplantation and main concern arise that MR people lacks cognitive skills to comply with complex post-operative procedures and medication regimens. Altogether, MR people are not considered for transplant operations as they were supposed to be incompetent and cannot decide autonomously. Conversely, a mentally challenged individual was selected as an organ donor, whereas, on the other side they cannot receive organ transplantation, for the reason that they are considered incompetent and cannot make independent decisions. In this situation an ethical dilemma was placed, where a MR individual was selected as an organ donor candidate as a result of surrogate's decision. At this moment, situation arises that when we practice taking consents, we practically, forget the patient's autonomy and our focus shifts from patient to family members. In some cases when the person is child or is unable to consent then surrogates may take decisions for them [5]. To make neutral decisions by the surrogate in consent procedure without exploitation of the rights of vulnerable people is of foremost considerations. 
Page 2 of 2

\section{Counter Argument}

In local context, involvement of family members in decision making process is a common practice a result of extended family system. More or less, this chain of command is not harmful and it often overrides individual decisions. However, the minute when it comes in to health care setting, from Pakistani patriarchal perspective giving the right to the family members to take decisions is in the best interest of the individual. Moreover, it can be argued that possibly the life of rest of family members is depending on that person who is sick and is transplant candidate. Subsequently, after internalizing the picture from 'utilitarian' perspective MR involvement in organ donation might be argued as maximization of happiness for family through minimizing the total harm.

\section{Justification of My Position}

In this situation, my position pertained to be more ethically acceptable in the favor of vulnerable people's rights. In Pakistani context though, family plays an essential role in making decisions in the best interest of individual, but taking away one's right is also not ignorable. In order to prevent rights of vulnerable people, involvement of ethical consultations in informed consent process is of great attention. As well involvement of incompetent individual in donation procedure on surrogate decision might be of great weigh with respect to happiness for maximum. One dimension of theory of distributive justice also supports this stance as; 'goods should be provided to those who are in most need' [6].

\section{Conclusion and Recommendations}

Free informed consent is generally considered as legal and ethically sound, but assessment of incompetent or vulnerable person is not an easy process. Thus, involvement of an incompetent person in organ donation seems to be trembling of their rights and justice to them. So selecting a surrogate who will take decisions without being bias is of great attention. Health care professionals, organizations and government need to step up so that ethically sound decisions could be made according to our context. Ethically sound laws and policies should be developed and implemented so that transplant candidates may receive organs without exploiting the rights of vulnerable people.

\section{References}

1. (1992) The ICD-10 Classification of mental and behavioural disorders: Clinical descriptions and diagnostic guidelines. World Health Organization, Geneva.

2. Chima SC (2013) Evaluating the quality of informed consent and contemporary clinical practices by medical doctors in South Africa: An empirical study. BMC Medical Ethics 14: S3.

3. Beauchamp T, Childress J (2001) Principles of biomedical ethics (7th edn.), Oxford University Press, New York.

4. Organ S (2000) Consensus statement on the live organ donor. JAMA 284: 2919-2926.

5. Chima SC (2013) Evaluating the quality of informed consent and contemporary clinical practices by medical doctors in South Africa: An empirical study. BMC Medical Ethics 14: S3.

6. Burkhardt M, Nathaniel A (2002) Ethics and issues in contemporary nursing (2nd edn.), Cengage Learning, Australia. 